\title{
Turnover and Retention Strategies Among Mental Health Workers Debbie L. Youngdoctor of Business Administrationtrophy Club, Texas Debyoungwahu@yahoo.com, (254) 421-41340rcid Id Https://orcid.org/0000-0001-6073-8160
}

Debbie L Young ( $\nabla$ debyoungwahu@yahoo.com )

Doctor of Business Administration Trophy Club

\section{Research}

Keywords: community mental health workers, turnover, retention, healthcare administrators, healthcare management

Posted Date: April 29th, 2021

DOl: https://doi.org/10.21203/rs.3.rs-465296/v1

License: (c) (i) This work is licensed under a Creative Commons Attribution 4.0 International License. Read Full License 


\section{Abstract}

\section{Purpose}

The purpose of this study was to explore retention strategies that healthcare administrators use to retain mental health workers in community mental health clinics. The study was guided by the question What strategies do HCAs use to retain their MHWs with more than 2 years of experience?

\section{Methods}

A qualitative case study analysis was performed. Interviews were conducted with six healthcare administrators at Fort Worth area community mental health clinics. Interviews were coded using thematic analysis. To increase the study's reliability and validity, the interview guide was field checked by experts and member checking performed on interview transcripts.

\section{Results}

The healthcare administrators identified six strategies for retention: providing good benefits packages, promoting worker health and wellness, providing competitive salaries, giving workers flexible scheduling options, incentivizing workers with rewards and positive reinforcement, and maintaining open communication channels.

\section{Conclusions}

The results from this study have positive implications for mental health workers, patients, and community mental health clinics. The strategies identified in this study can be implemented to increase worker satisfaction and reduce turnover. In doing so, patients will receive higher quality care and organizations will be more attractive to prospective employees.

\section{Introduction}

Mental health workers experience high turnover rates of more than 50\% (SAMHSA, 2017), a factor that has been linked to inadequate quality of care for patients in community mental health clinics (Bukach, Ejaz, Dawson, \& Gitter, 2017). Aside from contributing towards poor quality patient care, high turnover rates among mental health workers impose significant financial burdens on organizations (Cloutier, Felusiak, Hill, \& Pemberton-Jones, 2015) and increase worker dissatisfaction (Khamisa, Oldenburg, Peltzer, \& Ilic, 2015). Turnover is related to a number of factors such as low salary, poor work-life balance, relationship conflicts, and limited opportunities for career growth (Guha and Chakrabarti, 2016; WillardGrace et al., 2019). Since high turnover rates among mental health workers can lead to lower quality care, it is essential that health care administrators develop and implement effective retention strategies.

\section{Turnover and Retention in Healthcare}


Since mental health workers work closely with patients to develop trust and deliver high-quality care, it is essential that turnover be reduced. A recent report by the Substance Abuse and Mental Health Administration (2017) indicated that factors such as understaffing, increasing workloads, and a lack of training contributed towards turnover among mental health workers. High turnover rates disrupt the workplace cultures of mental health organizations and can have negative consequences at the organizational and individual level. For instance, organizations that experience high turnover might have difficulty with recruiting skilled professionals (Acker, 2018; Kim \& Fernandez, 2016), and incur significant costs associated with recruitment and training of new employees (Cameron, Sadlo, Hart, \& Walker, 2016). As a result, clinics with high turnover may find it hard to provide high-quality care in the future. Since many workers leave their jobs out of dissatisfaction, effective retention strategies include those that focus on improving employee motivation, workplace satisfaction, and employee engagement (Allen, Peltokorpi, \& Rubenstein, 2016).

\section{Employee Job Satisfaction and Dissatisfaction}

Employee satisfaction is driven by factors such as workplace culture, available resources, and satisfaction with the work itself (Kok, Herrell, Grossman, West, \& Wilk, 2016; Read \& Laschinger, 2015). Workplace satisfaction is driven by intrinsic motivation factors such as the opportunity for personal achievement, recognition, and the difficulty of the work (Al-Hamdan, Manojlovich, \& Tanima, 2016). Dissatisfaction with work is most often associated with extrinsic motivation factors including workplace relationships, salary, and benefits (Al-Hamdan et al., 2016). Within the mental healthcare industry, workers who have the most direct patient contact, such as nurses, tend to experience the lowest job satisfaction (Grossman-Kahn, Schoen, Mallett, Brentani, Kaselitz, \& Heisler, 2017). In organizational settings that are highly dependent on teamwork, such as mental healthcare, high turnover can result in increased workloads on the remaining team members, which can increase job dissatisfaction (Khamisa et al., 2015). Mental health workers also often work in stressful work environments, a factor which can further increase their job dissatisfaction (Richardson, 2017).

\section{Recruitment and Retention}

Effective retention strategies capitalize on the factors which motivate workers to join and stay at an organization. Factors that motivate mental health workers to join the field include the desire to care for patients in need (Garcia, McGeary, Finley, Ketchum, McGeary, \& Peterson, 2015). Others may seek to reduce the stigma associated with mental illness through education and awareness about possible causes and treatments (Grubbs, Fortney, Dean, Williams, \& Godleski, 2015). Workers who believe that they are fulfilling these goals are more likely to stay at their jobs. Workplace retention among mental health workers is also influenced by positive relationships with co-workers; the team delivery of healthcare and a shared understanding of human behavior often serve to form positive connections between mental health workers (Grubbs et al., 2015). On the contrary, factors such as small staff-to-patient ratios may serve to decrease retention among community mental health workers (Liu, Jack, Piette, Mangezi, Machando, \& Rwafa, 2016). The high-risk environment in which mental health workers work, coupled with 
inadequate pay, may also increase employee turnover (Grubbs et al., 2015). A lack of positive feedback or recognition (Holmberg, Sobis, \& Carlström, 2015), alongside a perceived lack of opportunity for career advancement (Beidas, Marcus, Wolk, Powell, Aarons, Evans, \& Babbar, 2015) also influence turnover rates among mental health workers.

\section{Purpose of the Study}

Recognizing that research on possible retention strategies for mental health workers is limited despite the burden high mental health worker turnover imposes on patients, employees, and organizations, the purpose of this study is to explore multiple retention strategies healthcare administrators use to retain their mental health workers with more than two years of experience in Texas community mental health clinics. The research question guiding the study is: What strategies do healthcare administrators use to retain their MHWs with more than 2 years of experience? For the purposes of this study, health care administrators (HCAs) were defined as nonclinical professionals who make decisions related to the dayto-day administrative operations of health care facilities and hospitals and ensure adherence to healthcare laws and regulations (Guo, Berkshire, Fulton, \& Hermanson, 2019; Hahn, Steinhäuser, \& Goetz, 2020). Mental health workers were those who provide treatment, documents care, and meet the primary health care needs of patients with mental illness or behavioral problems (Herschell, Kolko, Hart, Brabson, \& Gavin, 2020).

\section{Methods}

A qualitative case study design was selected for this study. Since the goal of this study was to develop an in-depth understanding of a specific phenomenon (in this case, strategies for retaining mental health workers), a qualitative methodology was the most appropriate (Anderson, 2017). While quantitative methods can be fruitful ways to developing more generalizable conclusions, such an approach is typically useful when the purpose of the research is to investigate a relationship between two or more variables acting on a large sample population (Antwi \& Hamza, 2015). Since this study involved conducting a small-scale investigation into how a certain phenomenon happens, qualitative methods were more appropriate. Furthermore, a case study design was selected so that the retention strategies employed by HCAs at multiple locations could be investigated. Other popular qualitative designs, such as ethnography and phenomenology, emphasize in-depth investigations of specific cultures (Goodson \& Vassar, 2011) or meanings behind lived experiences (Towers, Abushaikha, Ritchie, \& Holter, 2020), and so were not appropriate.

\section{Recruitment Procedure and Participants}

The participants in this study were recruited using purposive sampling from community mental health clinics in Fort Worth, Texas. Eligibility criteria were that participants had to be healthcare administrators with more than two years of experience with hiring and retaining mental health workers within their clinics. Healthcare administrators were chosen as the target population because they have the ability to implement new retention policies and are responsible for overseeing mental health workers. Approval was 
obtained from the Walden University Institutional Review Board (IRB). Initially, the head of Human Resources at community mental health clinics in Denton and north Tarrant counties were contacted to identify the appropriate person to obtain authorization for conducting the study. Personal invitations were emailed to identified HCAs with information about the purpose, methods, and potential benefits of the study. An informed consent document was also included in the emails. Interviews were only scheduled once administrators replied to the email with their consent within a 7-day period. Of the 11 interested volunteers, six satisfied the eligibility criteria. Since the goal of case study analysis is to collect in-depth data about a specific phenomenon (Yin, 2018), six participants were deemed an acceptable sample size. Interviews were guided by an interview protocol that followed a set of 7 pre-determined questions (see Appendix A). Three days after the interviews, interview summaries were prepared and sent to participants for member checking (Birt, Scott, Cavers, Campbell, \& Walter, 2016).

\section{Data Analysis Procedure}

Member checked interview summaries were analyzed with the aid of NVivo 12 software. Data was analyzed using thematic analysis, a technique used in qualitative studies used to generate themes based on careful analysis of interview data (Braun \& Clarke, 2019). The phases of thematic analysis include familiarization, coding, initial theme development, theme revision, theme finalization, and report generation (Braun \& Clarke, 2019). During the familiarization phase, interview transcripts were read several times to identify key words and phrases related to the research question. Codes were then used to describe these key words and then grouped together to generate the initial themes. Themes were revised to form more concise thematic groupings before the final list of themes was developed.

\section{Reliability and Validity}

In qualitative research, the researcher must ensure that the study's credibility, transferability, dependability, and confirmability are upheld (Lincoln \& Guba, 1985). In this study, an audit was used to increase dependability. The interview guide was tested by Human Resources professionals with more than 10 years of experience with implementing successful retention strategies to ensure that the interview questions were designed to answer the research question. Interviews were also member checked by participants to enhance credibility (Birt, Scott, Cavers, Campbell, \& Walter, 2016). Detailed methods procedures help uphold the transferability of this study by making it easier for future researchers to replicate this study or apply its findings in another context (Lincoln \& Guba, 1985). Confirmability was maximized by reducing bias through the use of a validated interview instrument and assessing the potential sources and contributions of researcher bias throughout the study process.

\section{Results And Discussion}

Participants discussed a variety of different retention strategies that their organizations used to improve worker satisfaction and retention. These strategies are summarized in Table 1 below: 


\begin{tabular}{|ll|}
\hline Strategy & Number of Responses \\
\hline Providing good benefits packages & 6 \\
\hline Promoting the health and wellness of mental health workers & 5 \\
\hline Providing competitive salaries & 3 \\
\hline Providing flexible scheduling options & 5 \\
\hline Incentivizing workers with rewards and positive reinforcement & 4 \\
\hline Open communication between workers and administrators & 4 \\
\hline
\end{tabular}

Providing good benefits packages helps retain employees. All of the participants identified good benefits packages as a successful retention strategy. When participants were asked about strategies, they have adopted to increase worker retention, four explicitly referenced their organization's benefits package. For example, Participant 6 stated, "We offer competitive benefits and salary packages including flexible schedules when possible." The other two participants implicitly referred to their organization's benefits as helping retain workers, with participant 4 explaining, "we offer school loan repayments, paid time off (PTO), if needed, time extended for patients' treatments, flexible schedules." Other empirical research has also highlighted the importance of good benefits packages in retaining employees, with Read \& Laschinger (2015) claiming that employee benefits may determine whether a business retains or loses its seasoned employees to their competitors who may offer more attractive benefits and salaries.

Providing competitive salaries helps retain employees. Half of the participants claimed that competitive salaries compensation helped retain workers. It is important that employees receive a salary that reflects their station and is competitive with that of similar positions at other organizations (Ghaffari et al., 2017). For some workers, a fair salary may outweigh other aspects of their job that are dissatisfactory (Wilson, 2015). If workers do not feel that they are fairly rewarded for their work, they may be more likely to leave, even if the organization offers other strong benefits. Therefore, it is essential that HCAs ensure their mental health workers are receiving fair and competitive salaries.

Promoting employee health helps retain employees. Another common retention strategy identified by participants was providing support and resources to workers experiencing work-related stress. Five of the six HCAs interviewed said that mental health and wellness strategies were used to improve worker satisfaction and retention. This is especially important in the mental healthcare field, where the stressful nature of the work can take a negative toll on the mental health of the workers (Grossman-Kahn et al., 2017). Participant 1 recognized that sometimes mental health workers need time to take a break by stating that "even though they are mental health professionals, these workers also need mental health days off when they are feeling overwhelmed." Participant 5 emphasized that their company provides multiple communication channels to mental health workers to help them cope with stressful situations. These responses demonstrated that HCAs are aware that their workers' mental health can be impacted by 
the stressful nature of their jobs, and that stress-relieving strategies are a commonly used tool to increasing worker retention.

Flexible scheduling options helps retain employees. Five participants highlighted providing employees with flexible schedules or sufficient paid time-off as a strategy for improving worker satisfaction and retention. Four participants used the exact phrase "flexible schedule" when describing their worker retention strategies. However, it is important to note that flexible schedules are just one of many possible incentives that can be provided to mental health workers. Other motivators such as salary and positive workplace culture can also help contribute to increased worker satisfaction and retention (Matei \& Abrudan, 2016). Other research has identified time-off, employee awards, mentoring programs, and promotions as strategies for targeting internal factors to increase worker retention.

Incentives may help to retain employees. Four participants listed incentives or bonuses as another strategy for retaining employees. Two of the four participants described how they provide yearly bonuses to employees, while the other two participants did not mention whether their bonuses were offered at certain intervals. This finding aligns with those of Read and Laschinger (2015), who demonstrated that the benefits offered to employees are critical to retention efforts. Organizations that do not offer such benefits risk losing their experienced employees to competitors that are willing to offer a more substantial compensation package. Bonuses and other incentives also present a more feasible retention option to organizations that cannot afford to increase the salary of all of their workers.

Open communication helps retain employees. Maintaining clear and open communication was another strategy repeatedly referenced by participants, with four of the six participants highlighting the important role that open communication channels between workers and administrators play in worker retention. As a result, workers are able to receive feedback and provide feedback to leadership. Willard-Grace et al. (2019) previously identified that a lack of employee engagement through administrative indifference could lead to work dissatisfaction among seasoned employees. By providing an outlet for workers to express their opinions and engage with leadership, organizations hope to prevent worker dissatisfaction. Sinha and Thaly (2013) offered similar suggestions, arguing that poor engagement among seasoned employees may result from poor communication or limited opportunities for worker involvement in decision-making.

\section{Implications and Recommendations}

The strategies for mental health worker retention identified in this study may be employed by HCAs in other clinics to increase the satisfaction and retention of their employees. Across the country, mental health worker retention rates have been estimated to be as high as 50\% (SAMHSA, 2017), highlighting the urgency with which successful retention strategies need to be implemented. An inability to retain mental health workers negatively impacts the facilities that demand MHWs and their services. Additionally, since high turnover rates are associated with inadequate patient care for patients at community mental health clinics, the results of this study have positive implications with regards to improving patient care (Bukach et al., 2017). By understanding and implementing effective retention strategies, the findings of this study 
may also help organizations increase the number of qualified and experienced MHWs, improving the quality of mental health services they offer. The retention strategies identified in this study also have positive implications for mental health workers themselves, as participants in this study reported their workers are more satisfied with their jobs.

The retention strategies discussed in this study should be implemented based on an assessment of current retention strategies and job satisfaction levels of mental health workers. Based on current workplace policies and worker satisfaction, administrators may choose to offer more flexible scheduling options, enhanced compensation packages, or incentives to support the satisfaction of mental health workers. Alternatively, administrators may also choose to adopt stress reduction practices in order to enhance the health and wellness of mental health workers, thereby reducing job dissatisfaction and preventing occupational burnout. Upper administration of organizations should encourage administrators, particularly those who are involved in hiring, managing, and engaging with workers within the mental health field, to consider the potential implications of the current study's findings, conclusions, and recommendations.

\section{Limitations}

There were several limitations to this study. Since this study had a sample population of only six HCAs working in the same two counties, it is difficult to generalize the findings to organizations outside of the Fort Worth area. While data gathered from the interviews enabled the development of an in-depth understanding of certain factors that influence mental health worker retention, it certainly did not capture all of the factors related to worker retention. Additionally, it is not possible to make statements of causality, correlation, or prediction from qualitative methods alone. For instance, while this study demonstrated that flexible scheduling options can help improve worker satisfaction and retention, it is not clear if a lack of flexibility is a cause of worker dissatisfaction and attrition. Therefore, future research on retention strategies used to retain mental health workers should integrate quantitative methodologies to investigate questions of significance, causality, and correlation. Quantitative studies also typically deal with larger sample sizes, meaning the results are more generalizable. Future research should also consider including HCAs who work in different healthcare settings. Mental health workers who work in inpatient hospital settings experience a different work environment than workers who work in community clinics; therefore, it is likely that worker satisfaction and retention is influenced by different factors. Investigating how HCAs can improve the retention of mental health workers across a variety of care settings can contribute to the growth and development of the mental healthcare field more broadly.

\section{Conclusion}

Healthcare administrators can implement several strategies to increase the well-being and retention of mental health workers at community clinics. Such strategies include providing good benefits packages, promoting worker health and wellness, providing competitive salaries, giving workers flexible scheduling options, incentivizing workers with rewards and positive reinforcement, and maintaining open 
communication channels. Doing so will help mental health clinics deliver higher quality patient care, leading to improved patient outcomes. Additionally, these retention strategies will help increase worker satisfaction and lead to the development of a more positive workplace culture, making the organization more attractive to prospective employees. While the results of this study provide important insights into how retention of mental health workers can be improved, more research across different healthcare settings is needed to determine what factors cause worker dissatisfaction and attrition so that more comprehensive retention strategies can be developed.

\section{Declarations}

\section{Ethics approval and consent to participate.}

Not applicable

\section{Consent for publication}

Not applicable

\section{Availability of data and materials}

The datasets generated and/or analyzed during the current study are available in the ProQuest repository, Strategies to Retain Mental Health Workers in Community Mental Health Clinics - ProQuest. The datasets used and/or analyzed during the current study are available from the corresponding author on reasonable request.

\section{Competing interests}

None to declare.

\section{Funding}

My journal is being funded solely by myself as the author. I do not have any sponsors or donors.

\section{Authors' contributions}

The study's findings will enhance mental health administrators' awareness of based on the recommended practices and policies, which other HCAs have implemented or adopted to increase employee retention in their organizations. The insights derived from this research process represents both effective and ineffective retention strategies by addressing high employee turnover among mental health workers.

\section{Acknowledgements}

Not applicable 


\section{Authors' information (optional)}

A recent doctoral graduated with a human resources background of over 30 years, with ten years being in the health administration industry. I plan to enter the academic field in the next few years. I want to give back to both fields, which have given me a great career. At the same time, I know that the competition is fierce in the academic world, so I believe in getting my article published not only in any journal. A journal aligned in my field would allow me to advance in the academic world and provide knowledge to the field. I have not potential issues with the journal's policies, and I abide by the policies forthwith.

\section{References}

1. Acker, G. (2018). Self-care practices among social workers: Do they predict job satisfaction and turnover intention? Social Work in Mental Health, 16(6), 1-15. doi:10.1080/15332985.2018.1494082

2. Al-Hamdan, Z., Manojlovich, M., \& Tanima, B. (2016). Jordanian nursing work environments, intent to stay, and job satisfaction. Journal of Nursing Scholarship, 49, 103-110. doi:10.1111/jnu.12265

3. Allen, D., Peltokorpi, V., \& Rubenstein, A. (2016). When "embedded” means "stuck": Moderating effects of job embeddedness in adverse work environments. Journal of Applied Psychology, 101, 1670. doi:10.1037/apl0000134

4. Anderson, V. (2017). Criteria for evaluating qualitative research. Human Resource Development Quarterly, 28(2), 125-133. doi:10.1002/hrdq.21282

5. Antwi, S. K., \& Hamza, K. (2015). Qualitative and quantitative research paradigms in business research: A philosophical reflection. European Journal of Business and Management, 7, $217-225$. Retrieved from https://www.iiste.org/

6. Beidas, R., Marcus, S., Wolk, C., Powell, B., Aarons, G., \& Evans, A., \& Babbar, S. (2015b). A prospective examination of clinician and supervisor turnover within the context of implementation of evidencebased practices in a publicly-funded mental health system. Administration and Policy in Mental Health and Mental Health Services Research,43, 640-649. doi:10.1007/s10488-015-0673-6

7. Birt, L., Scott, S., Cavers, D., Campbell, C., \& Walter, F. (2016). Member checking: A tool to enhance trustworthiness or merely a nod to validation?Qualitative Health Research, 26, 1802-1811. doi:10.1177/1049732316654870

8. Braun, V., \& Clarke, V. (2019). Reflecting on reflexive thematic analysis. Qualitative Research in Sport, 17(4), 589-597. doi:10.1080/2159676X.2019.1628806

9. Bukach, A. M., Ejaz, F. K., Dawson, N., \& Gitter, R. J. (2017). Turnover among community mental health workers in Ohio.Administration and Policy in Mental Health and Mental Health Services Research, 44, 115-122. doi:10.1007/s10488-015-0706-1

10. Cameron, J., Sadlo, G., Hart, A., \& Walker, C. (2016). Return-to-work support for employees with mental health problems: Identifying and responding to key challenges of sick leave. British Journal of Occupational Therapy, 79, 275-283. doi:10.1177/0308022615627176 
11. Cloutier, O., Felusiak, L., Hill, C., \& Pemberton-Jones, E. (2015). The importance of developing strategies for employee retention. Journal of Leadership, Accountability and Ethics, 12(2), 119-129. Retrieved from http://www.na-businesspress.com/jlaeopen.html

12. Garcia, H., McGeary, C., Finley, E., Ketchum, N., McGeary, D., \& Peterson, A. (2015). Burnout among psychiatrists in the Veterans Health Administration.Burnout Research, 2(4), 108-114. doi:10.1016/j.burn.2015.10.001

13. Ghaffari, S., Shah, I., Burgoyne, J., Nazri, M., \& Aziz, J. (2017). Research paper: Investigating the mediation role of respect for employees on the relationship between participative leadership and job satisfaction: A case study at Universiti Teknologi Malaysia. Australian Journal of Basic and Applied Sciences, 11(1), 1-13. Retrieved from http://www.ajbasweb.com/

14. Goodson, L., \& Vassar, M. (2011). An overview of ethnography in health care and medical education research. Journal of Education Evaluation for Health Professions, 8(4), 1-5. doi:10.3352/jeehp.2011.8.4

15. Grossman-Kahn, R., Schoen, J., Mallett, J., Brentani, A., Kaselitz, E., \& Heisler, M. (2017). Challenges facing community health workers in Brazil's Family Health Strategy: A qualitative study. International Journal of Health Planning and Management, 33, 309-320. doi:10.1002/hpm.2456

16. Grubbs, K., Fortney, J., Dean, T., Williams, J., \& Godleski, L. (2015). A comparison of mental health diagnoses treated via interactive video and face to face in the Veterans Health care Administration. Telemedicine And E-Health, 21, 564-566. doi:10.1089/tmj.2014.0152

17. Guha, S., \& Chakrabarti, S. (2016). Differentials in attitude and employee turnover propensity: A study of information technology professionals. Global Business and Management Research, 8(1), 1-17. doi:10.1177/0972150916631086

18. Guo, R., Berkshire, S. D., Fulton, L. V., \& Hermanson, P. M. (2019). Predicting intention to use evidencebased management among US health care administrators: Application of the theory of planned behavior and structural equation modeling. International Journal of Health Care Management, 12(1), 25-32. doi:10.1080/20479700.2017.1336856

19. Hahn, K., Steinhäuser, J., \& Goetz, K. (2020). Equity in health care: A qualitative study with refugees, health care professionals, and administrators in one region in Germany. BioMed Research International, 2020, 1-8. doi:10.1155/2020/4647389

20. Herschell, A. D., Kolko, D. J., Hart, J. A., Brabson, L. A., \& Gavin, J. G. (2020). Mixed method study of workforce turnover and evidence-based treatment implementation in community behavioral health care settings. Child Abuse \& Neglect, 102, 104419. doi:10.1016/j.chiabu.2020.104419

21. Holmberg, C., Sobis, I., \& Carlström, E. (2015). Job satisfaction among Swedish mental health nursing staff: A cross-sectional survey. International Journal of Public Administration, 39, 429-436. doi:10.1080/01900692.2015.1018432

22. Khamisa, N., Oldenburg, B., Peltzer, K., \& llic, D. (2015). Work-related stress, burnout, job satisfaction and general health of nurses. International Journal of Environmental Research and Public Health, 12, 652-666. doi:10.3390/ijerph120100652 
23. Kim, S., \& Fernandez, S. (2016). Employee empowerment and turnover intention in the U.S. Federal Bureaucracy. American Review of Public Administration, 47(1), 4-22. doi:10.1177/0275074015583712

24. Kok, B., Herrell, R., Grossman, S., West, J., \& Wilk, J. (2016). Prevalence of professional burnout among military mental health service providers. Psychiatric Services, 67(1), 137-140. doi:10.1176/appi.ps.201400430

25. Lincoln, Y. S. \& Guba, E. G. (1985). Establishing trustworthiness. In Y. Lincoln \& E. Guba (Eds.), Naturalistic inquiry (pp. 289-327). Newbury Park, CA: SAGE.

26. Liu, G., Jack, H., Piette, A., Mangezi, W., Machando, D., \& Rwafa, C. (2016). Mental health training for health workers in Africa: A systematic review. The Lancet Psychiatry, 3(1), 65-76. doi:10.1016/s22150366(15)00379-x

27. Matei, M.-C., \& Abrudan, M.-M. (2016). Adapting Herzberg's two-factor theory to the cultural context of Romania. Procedia - Social and Behavioral Sciences, 221, 95-104. doi:10.1016/j.sbspro.2016.05.094

28. Read, E. A., \& Laschinger, H. K. (2015). The influence of authentic leadership and empowerment on nurses' relational social capital, mental health and job satisfaction over the first year of practice. Journal of Advanced Nursing, 71, 1611-1623. doi:10.1111/jan.12625

29. Richardson, K. M. (2017). Managing employee stress and wellness in the new millennium. Journal of Occupational Health Psychology, 22, 423-428. doi:10.1037/ocp0000066

30. Sinha, V., \& Thaly, P. (2013). A review on changing trend of recruitment practice to enhance the quality of hiring in global organizations. Management: Journal of contemporary management issues, 18(2), 141-156. Retrieved from https://www.proquest.com/docview/1476270650/abstract/52AB9CDB93F4677PQ/1

31. Substance Abuse and Mental Health Services Administration. (2017). Behavioral health workforce: Quality assurance practices in substance abuse treatment facilities. Retrieved from https://www.samhsa.gov/data/report/behavioral-health-workforce-quality-assurance-practicessubstance-abuse-treatment-facilities

32. Towers, N., Abushaikha, I., Ritchie, J., \& Holter, A. (2020). The impact of phenomenological methodology development in supply chain management research. Supply Chain Management, 25(4), 443-456. doi:10.1108/SCM-04-2019-0153

33. Willard-Grace, R., Knox, M., Huang, B., Hammer, H., Kivlahan, C., \& Grumbach, K. (2019). Burnout and health care workforce turnover. Annals of Family Medicine, 17(1), 36-41. doi:10.1370/afm.2338

34. Wilson, N. (2015). Factors that affect job satisfaction and intention to leave of allied health professionals in a metropolitan hospital. Australian Health Review, 39, 290. doi:10.1071/ah14198 35. Yin, R. K. (2018). Case study research and applications: Design and methods(6th ed.). Los Angeles, CA: SAGE.

\section{Supplementary Files}


This is a list of supplementary files associated with this preprint. Click to download.

- AppendixA.docx 